\title{
Dynamic of the States of Three Different Stock Markets from Correlation and Partial Correlation Changes
}

\author{
Shafiqul Alam¹, Nahid Akter², Mohammad Rubel Miah', Mohammed Javed Hossain², \\ Ashadun Nobi ${ }^{2, *}$ \\ ${ }^{1}$ Department of Business Administration, Noakhali Science and Technology University, Noakhali, Bangladesh \\ ${ }^{2}$ Department of Computer Science and Telecommunication Engineering, Noakhali Science and Technology University, Noakhali, Bangladesh \\ Email address: \\ shafiq.alam@nstu.edu.bd (S. Alam),nahid_akter@nstu.edu.bd (N. Akter), rubeldba@nstu.edu.bd (M. R. Miah), \\ javed.nstu@gmail.com (M. J. Hossain), ashad@nstu.edu.bd (A. Nobi) \\ ${ }^{*}$ Corresponding author
}

\section{To cite this article:}

Shafiqul Alam, Nahid Akter, Mohammad Rubel Miah, Mohammed Javed Hossain, Ashadun Nobi. Dynamic of the States of Three Different Stock Markets from Correlation and Partial Correlation Changes. International Journal of Business and Economics Research.

Vol. 10, No. 4, 2021, pp. 155-161. doi: 10.11648/j.ijber.20211004.16

Received: July 7, 2021; Accepted: July 21, 2021; Published: August 18, 2021

\begin{abstract}
The core focus of the study is to examine financial states using index effect on stock to stock correlations of developed, developing and emerging market. The three markets such as S\&P 500, KOSPI 200 and DSE are declared as developed, developing and emerging market respectively. To study the similarity between stock price changes, we calculate the time series of the daily log return. Closing stock prices of the targeted markets have been used to measure the daily return of the stocks. To analyze the market mobility, Pearson correlation coefficient, partial correlation, and index effect on stock to stock correlation techniques have been applied. The study found that the companies of developed and emerging market are more strongly correlated than those of developing market during big crash. On the other hand, developing market shows less index effect on stock correlations during crisis. Moreover, insignificant index effect has been found in emerging market during calm state. No significant effect of DSE index on stock to stock correlations in the period of global financial crisis has been observed, implying that global financial crisis did not hit to the DSE in this period. Before the market crash, the interactions between stocks became low enough which corresponds to lower value of average correlation for all types of market. Finally, the change of correlation and partial correlation can be a good indicator to identify and predict the financial states of all the markets which will further helps the stakeholders to make proper economic decisions.
\end{abstract}

Keywords: Stock-Stock Correlation, Partial Correlation, Stock-Index Correlation, Market Crash, Global Financial Crisis

\section{Introduction}

Financial market is a complex system where different techniques are used to understand the behavior and dynamics of financial markets [1]. The extraction of information from financial time series, correlation technique is persistently used that is useful for many practical applications such as portfolio optimization, risk management, and option pricing [2-7]. The time series describing stock returns, market index returnsand currency exchange rates have been investigated using correlation technique [2-15]. Correlation based networks such as threshold network, minimal spanning tree, planar maximally filtered graph are also used to investigate interactions across different financial agents $[2,10,13,16-$ 19]. The Pearson correlation is commonly used to indicate the level of similarity in the price changes for a given pair of stocks. But it is not used to measure whether other stocks influence the relationship between them. This technique has been introduced for the study of financial data extended and applied to other systems, such as the immune system and semantic networks [11-14].

The main goal of the study is to understand the dynamic of index effect on stock correlations which was investigated for developed, developing and emerging market of S\&P 500, KOSPI 200 and DSE respectively. A comparison among three markets helps to identify and predict the market state.

The rest of the paper is organized as follows: Section two 
discusses research methods that contain data collection, cross-correlation, partial correlation and index effect methods. Section three presents' results and discussion where section five concludes the paper with proposed definition to understand the market movement.

\section{Research Methods}

\subsection{Data Collection}

In this study, we have used closing stock price to analyze the USA stock market, Korean stock market and Dhaka stock market. The closing stock price is significant for several reasons. During working hour of a day, the stock prices of a company change almost in each second. The closing prices of stock indices give almost all information of the change of prices of a day. Investors, traders, financial institutions, regulators, stakeholders, economists and also the researchers use it as a reference point to determine performance over a specific time such as one year, a week and over a shorter time period such as one minute or less. In fact, investors and other stakeholders base their decisions on closing stock prices. Institutional investors monitor a stock's closing price to make decisions regarding their investment portfolios. The daily closing prices of 377 indices from the S\&P 500 between 2004 and 2012, 165 indices from the KOSPI 200 between 2004 and 2012, and 220 indices from the Dhaka Stock Exchange (DSE) between 2006 and 2012 were analyzed. These companies survived in the market during these periods. The one year time window and subsequently one month time window were considered.

For S\&P 500 and KOSPI 200, data have been collected from Yahoo finance from 2004 to 2012. In these periods, both markets have been affected by different kinds of crises that alter the hierarchy of the financial network. For example, there were the subprime mortgage crisis in 2007, global financial crisis in 2008, and European sovereign debt (ESD) crisis in 2011. For DSE, data have been collected from the archive of DSE from 2006 to 2012. In these periods, the market has been affected by severe crises that alter the hierarchy of the financial network. We have mainly considered the stock prices before, during and after the market crash, for this reason time period variation is arisen among S\&P 500, KOSPI 200 and DSE.

\subsection{Methodology}

\subsubsection{Cross Correlation}

The similarity between stock price changes is commonly calculated by the Pearson's correlation coefficient [20-22]. The raw stock correlations are calculated for time series of the $\log$ of the daily return [21-28] as

$$
r_{i}(t)=\log \left[P_{i}(t)\right]-\log \left[P_{i}(t-1)\right]
$$

where, $P_{i}(t)$ is the daily adjusted closing price of stock $\mathrm{i}$ at day t. The raw stock correlations are calculated using the Pearson's correlation coefficient $C(i, j)$ between every pair of stocks $\mathrm{i}$ and $\mathrm{j}[11]$ is

$$
C(i, j)=\frac{\left.\left\langle\left(r_{i}\right)-\left\langle r_{i}\right\rangle\right) \cdot\left(r_{j}-\left\langle r_{j}\right\rangle\right)\right\rangle}{\sigma_{i} \sigma_{j}}
$$

where, \langle\rangle denotes average, and $\sigma$ are the standard deviations (STD).

\subsubsection{Partial Correlation}

Recently, partial correlation has been playing an important role to indicate the financial state of a market [11, 20, 24]. To calculate the partial correlation, the index effect is not needed to be considered. The residual or partial correlation $\rho(i, j \mid m)$ between stocks $\mathrm{i}$ and $\mathrm{j}$, using the Index (m) as the mediating variable is defined by $[11,20,24]$

$$
\rho(i, j \mid m)=\frac{C(i, j)-C(i, m) \cdot C(j, m)}{\sqrt{\left(1-C^{2}(i, m)\right) \cdot\left(1-C^{2}(j, m)\right)}}
$$

where, $\rho(i, j \mid m)$, can be viewed as the residual correlation between stocks $i$ and $j$, after subtraction of the contribution of the correlation between each of the stocks with the Index.

\subsubsection{Index Effect}

The index effect on stock-stock correlations for the targeted markets and to identify the financial states of the market in a period $t$, we propose definition as

$$
\xi(t)=\left\langle C_{i j}(t)\right\rangle_{i j}-\left\langle\rho_{i, j \mid m}(t)\right\rangle_{i, j \mid m}
$$

where, $C_{i j}$ is the cross-correlation between the companies of $\mathrm{i}$ and $\mathrm{j}, \rho_{i, j \mid m}$ is the partial correlations after subtraction of index contribution on stock-stock correlations and \langle\rangle is the time average over all components. So, $\xi$ indicates the average index effect on stock-stock correlations in a period.

\section{Results and Discussion}

\subsection{Average Correlation with the Evolution of Time}

Figure 1 shows the overview of the change in cross correlation of S\&P 500 (Developed), KOSPI 200 (Developing) and DSE (Emerging market) from calm state to crisis state of the markets. It helps us to compare the state of the market at different times. Horizontal axis shows the time window (2004-2012) for one year gap in each block while vertical axis shows average stock-stock correlations. The higher correlation between stocks indicates the stiffness of the market while the lower value of correlation denotes the calm state. The average cross-correlation is lower for all the markets in the beginning of the analysis period which implies the soft state of the market. To observe the value of average correlation just before market crash is our aim. The lowest cross correlations are found before the market crash for different time period for the above mentioned markets. This result is consistent with the findings of the other studies [23, 29] Other researchers also observe that the average crosscorrelations increase during every crisis and drop after the crises. A remarkable point is that the average correlation is higher in developed and emerging market in the crises period which implies that the companies in developed and emerging market are more strongly correlated in the crisis time than 
those of developing market.

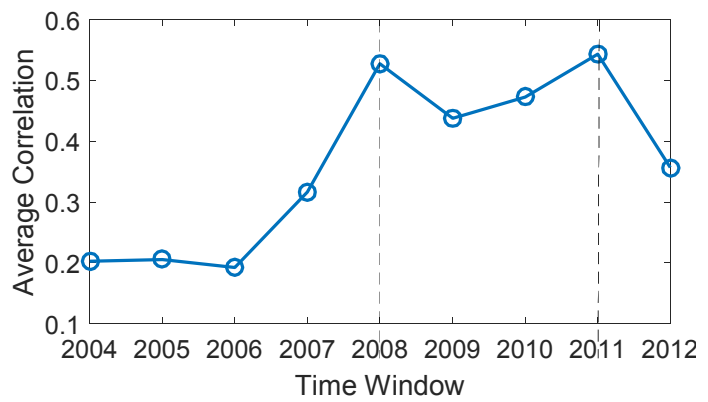

(a) S \& P 500

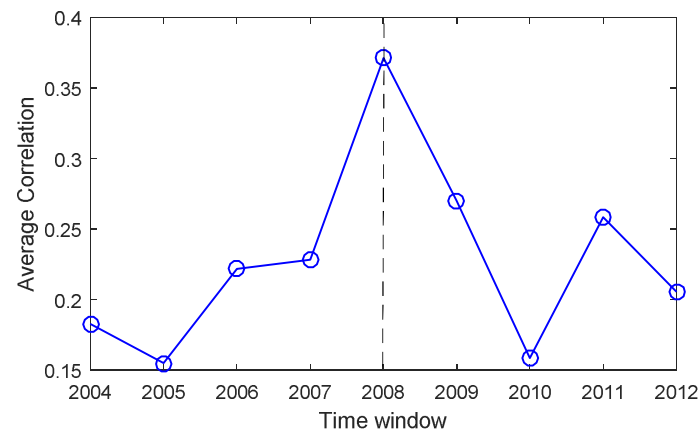

(b) KOSPI

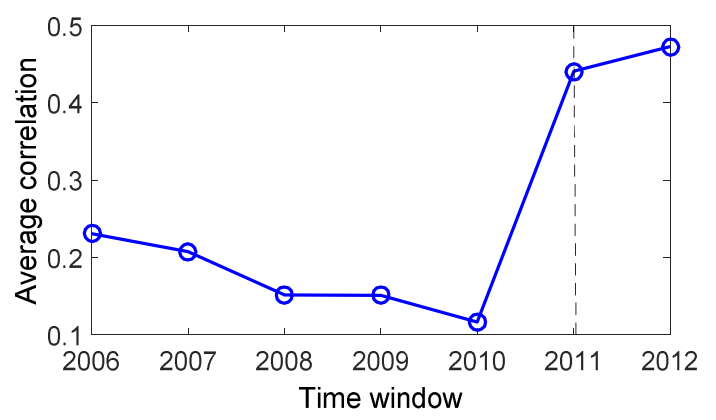

(c) DSE

Figure 1. Change of average correlation with the evolution of time.

The severe crisis was occurred in the US market and the Korean market in 2008 and DSE was in 2011. The global financial crisis did not hit to the DSE market. Before the market crash, the interactions between stocks became low enough which corresponds to lower value of average correlation for all types of market that can be a good indicator for upcoming crisis.

After temporarily stabilized in 2009, the average correlation of US market turned up in 2010 and 2011 due to European sovereign crisis. Again, US market became melt down in 2012 although the correlation level did not come back like the calm state of 2004. Observing low level of average correlation in 2004 and 2005, the calm state of the market, Korean market turned up and reached its climax in 2008 during global financial crisis. Then the average correlation sharply turned down in 2009 and 2010 while it turned up in 2011 during European debt crisis. On the other hand, after market crash of DSE in 2011, the highest average cross-correlation has been observed in 2012 which indicates that market is still under turbulent.

\subsection{Dynamism of Average Correlation and Partial Correlation}

The change of average correlation and the partial correlation with the one month time window from January, 2004 to December, 2012 for S\&P 500, KOSPI 200 and from 2006 to 2012 for DSE is shown in figure 2. It is showing index effect on stock-stock correlations before, during and after the periods of market crash. The study has found a little dynamicity of partial correlation in both developed and developing market but observing noteworthy dynamicity of partial correlation in emerging market.

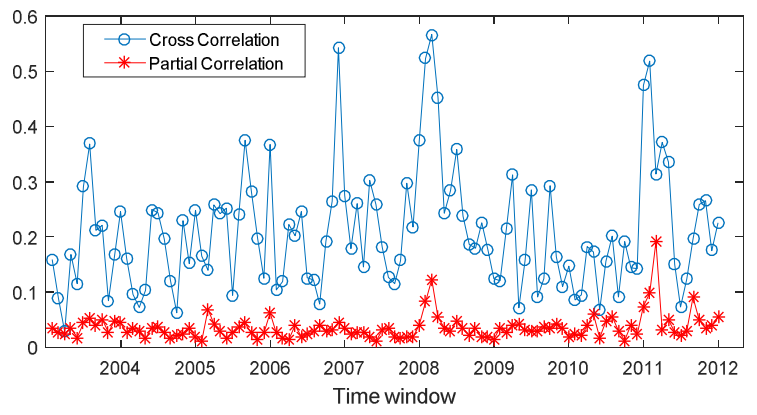

(a) S \& P 500

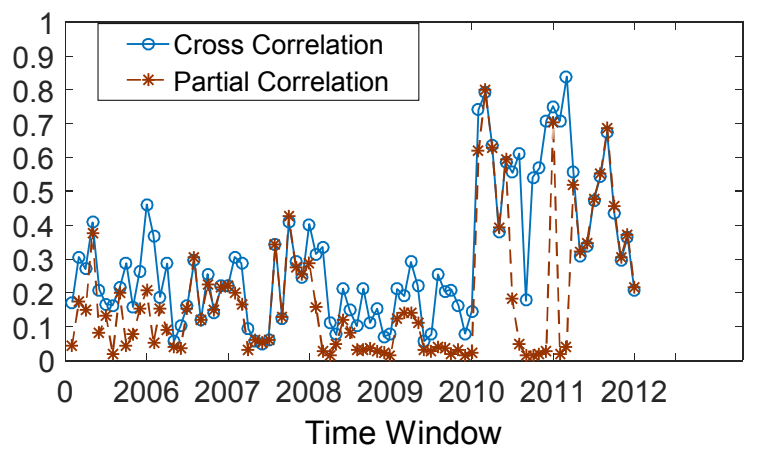

(b) KOSPI

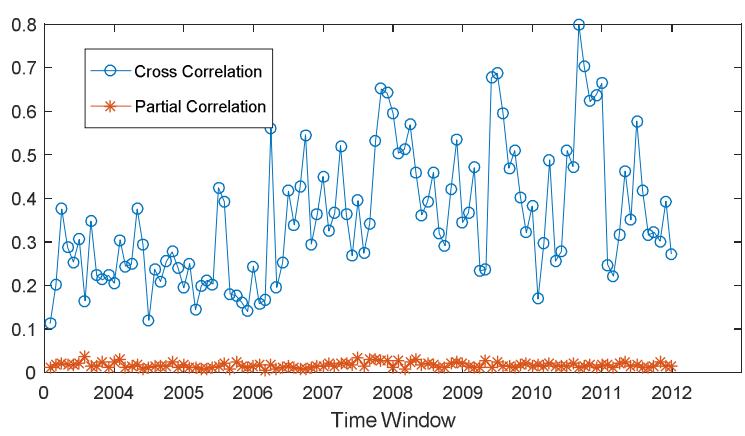

(c) DSE

Figure 2. Change of average correlation and partial correlation.

The study observed fluctuation of average cross correlation of both USA and Korean market in our observing period. However, the average partial correlation did not show significant fluctuation like average correlation 
in USA and Korean market. It implies a significant effect of index on stock correlation both USA and Korean market during the whole period. The result is consistent with the findings of previous studies [11, 20, 24]. After observing low index effect, a marked stiff has been observed in index effect during financial crises in the market S\&P 500 [11]. A strong similarity in the structure during the two crises has been observed, followed by consecutive quarters with low values of three rank correlations, representing the change in structure [20]. The index effect reached in extreme level during the crises. On the other hand, the trend of correlation and partial correlation is almost similar except few variations in different periods from 2006 to the mid of 2011 in DSE, but the remarkable variation has been observed from May 2011 to February 2012, just before and during the market crash.

It implies that the index effect on stock-stock correlations is high in these periods. No difference has been observed between correlation and partial correlation in some periods in DSE implying that there is no index effect on stock-stock correlations and market was calm state in that periods. The higher index effect on stock- stock correlations can be considered a signal that the stock market is going to vulnerable state. The trends of DSE market, where the variation of correlation and partial correlation for every year is clearly shown in figure 3.

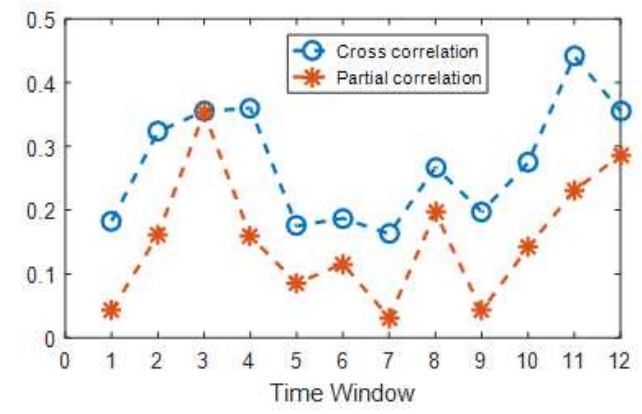

(a) 2006

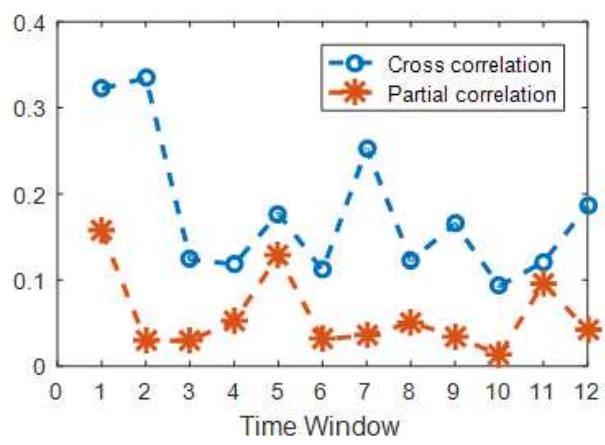

(d) 2009

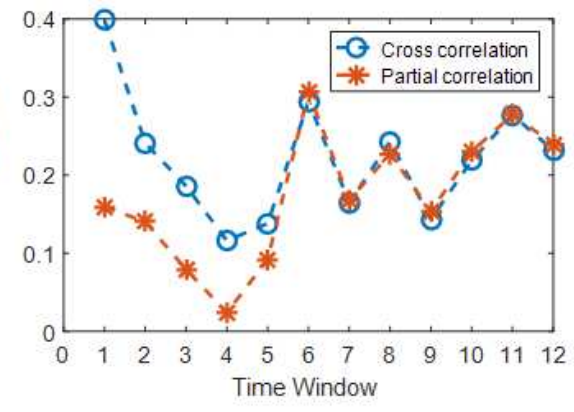

(b) 2007

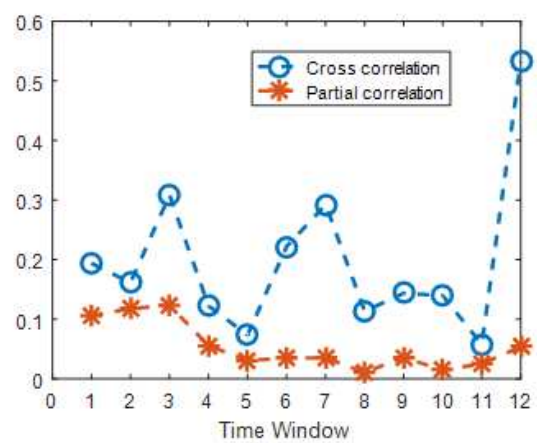

(e) 2010

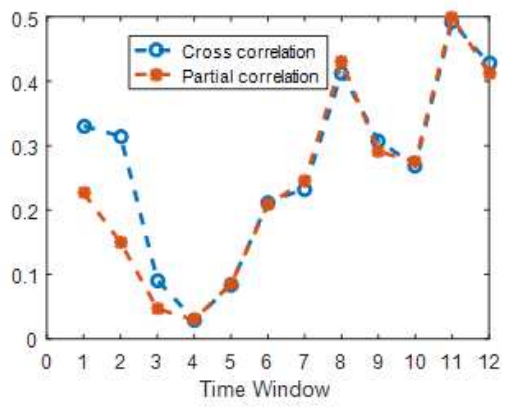

(c) 2008

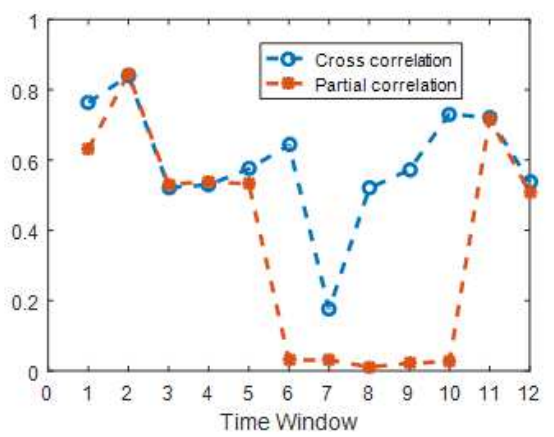

(f) 2011

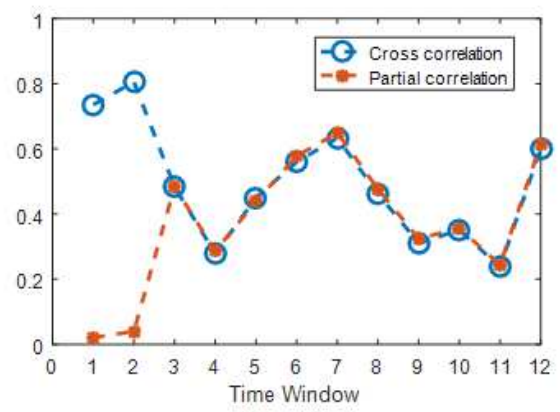

(g) 2012

Figure 3. Change of average correlation and partial correlation for DSE market.

It has been observed from the figure 3 that different months in different years had notable variation in correlation and partial correlation in DSE. Most of the time in 2006, the index effect on stock-stock correlations was low except September and December. The remarkable variation between correlation and partial correlation has been observed in the beginning of 2007 while observing the minimum or no variation during the rest time in that year as well as in 2008. From the very beginning of 2009, again the market looked on the variation between correlation and partial correlation during the whole year and it continued in the whole period of 2010 while having significant index effect in the last half of 
2010. During the last half of 2011 there was a gap between correlation and partial correlation and the variation reached its climax at the beginning of the first quarter of 2012. The rest of the period, there was no variation of correlation and partial correlation. These periods are traced as calm state. It implies that index effect on stock correlations during calm state is insignificant.

\subsection{Average Stock-Index Correlation}

The average stock-index correlations of developed, developing and local market are shown in figure 4 (a), (b) and (c) respectively over the one month time window for every nod. The higher values of average stock-index correlation of stock markets indicate strong stock-index interaction while lower values indicate the opposite.

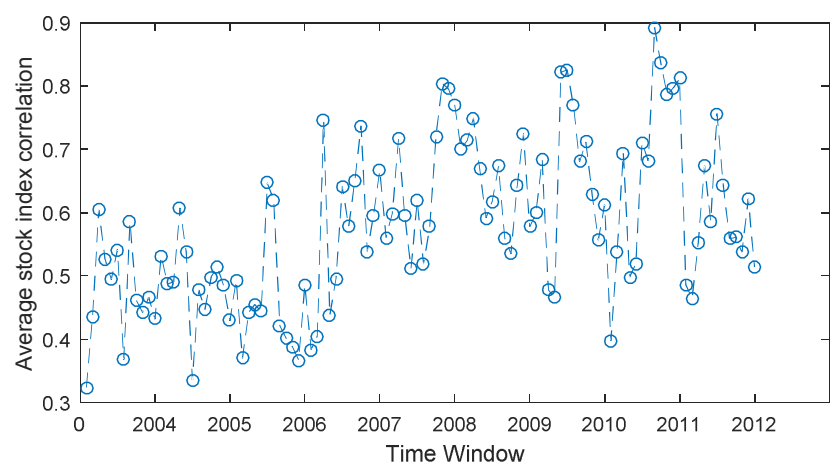

(a) S \& P 500

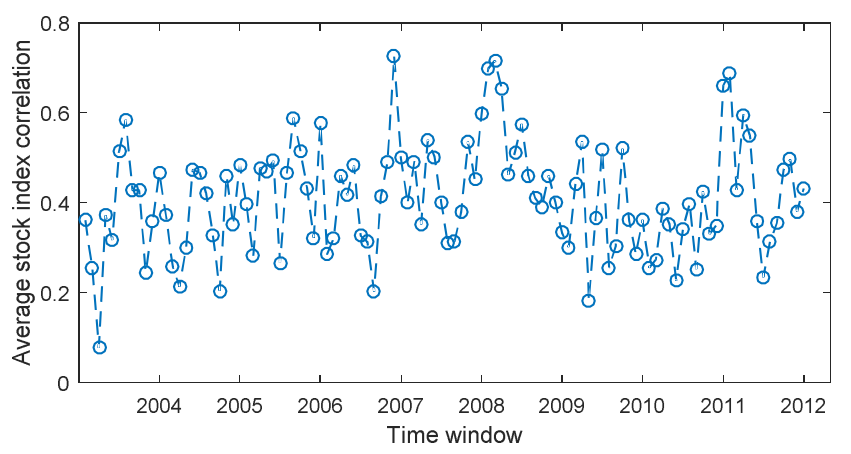

(b) KOSPI

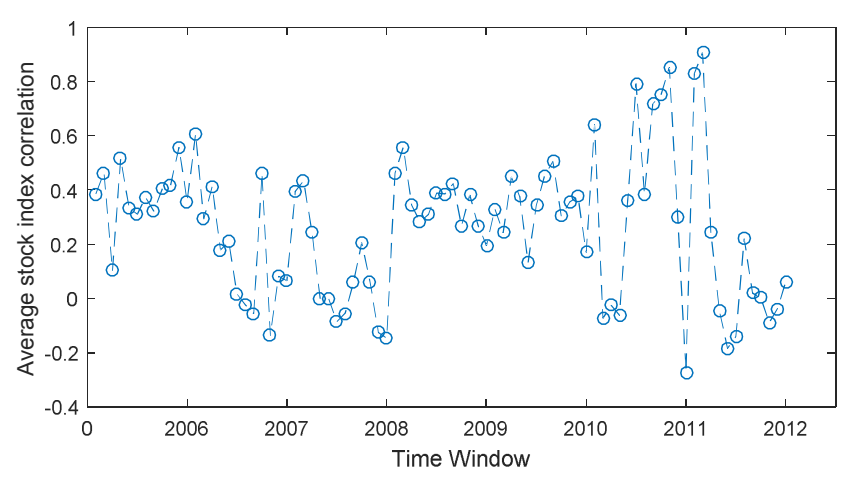

(c) DSE

Figure 4. Average stock-index correlation.

The average stock-index correlations of developed market are varying from 0.3 to 0.9 , from -0.25 to 0.9 for emerging market and from 0.1 and 0.75 of developing market. It indicates that the interactions between stock and index of developed and emerging market are higher than that of developing market. The most values of average stock-index correlation of both developing and emerging market is lower than 0.5 while for global market is greater than 0.5 . It emphasizes our previous result that the interactions of stockindex of the developed market are stronger than that of the other markets. The peak values of stock-index correlations are found during financial crises for all markets. It means that stock-index interactions are high during crises. The negative correlations between stock and index of emerging markets are found in some periods. It implies that there is no interaction between stock and index at those periods. We need more analysis in this regard which we will leave for future investigation.

\subsection{Financial States Using Index Effect}

The average index effect on stock-stock correlations $\xi$ in a period (from eq. 4) is found that changes notably before and during financial market crash. Therefore, knowing the change of $\xi$ with one month time window, one can identify the present state of the market.

Figure 5 shows the change of the state of all markets with the evolution of time. Each value is calculated by subtracting average partial correlation from average correlation in the same period. The more the value is, the more the index effect is on stock price. The red and deep red colored blocks represent severe impact of index effect on stock. This new representation gives a complete overview about the present state of the market which can be useful to predict the future state of the market. The pictorial presentation can give the overall picture of all markets around big crisis. In vertical axis we put the month whereas in horizontal axis we put the year. So the change of market states from one month to another month in a year can be understood by the change of color.

The low effect of index on stock-stock correlations are found from 2004 to 2005 for S\&P 500. The effect of index on stock-stock correlations have been increased in June and July of 2006. The remarkable effect of index on stock correlation is found in most of the periods of 2007 and 2008 just before and during global market crash. The index effects on stockstock correlation became high and reached at climax during financial crisis in 2008. After global financial crisis, the significant effect of index on stock-stock correlations has been also found in most of the periods from 2009 to 2011. It implies that after global financial crisis, market did not come back to calm state in 2009 and due to European sovereign debt crisis, the significant effect of index are found in most of the periods of 2010. The index effect has also been observed in several months in 2012 representing that market is still in the vulnerable state.

In KOSPI 200, the study observed the low index effect on stock correlations from January 2004 to September 2008 with few exceptions such as August 2006 and November 2007. 
The index effects turned up in October 2008 and went peak in the beginning of 2009 just after the global financial crisis. During crisis, we observe lower effect of index on stockstock correlations in KOSPI 200 than that of S\&P 500. After global financial crisis, the significant effect of index on stock-stock correlations has been turned down in most of the periods from May 2009 to November 2011. It implies that after global financial crisis, market came back to calm state. Again the remarkable effect of index on stock-stock correlation are found from November 2011 and continued up to April 2012 implying the effect of European sovereign debt crisis in the market.

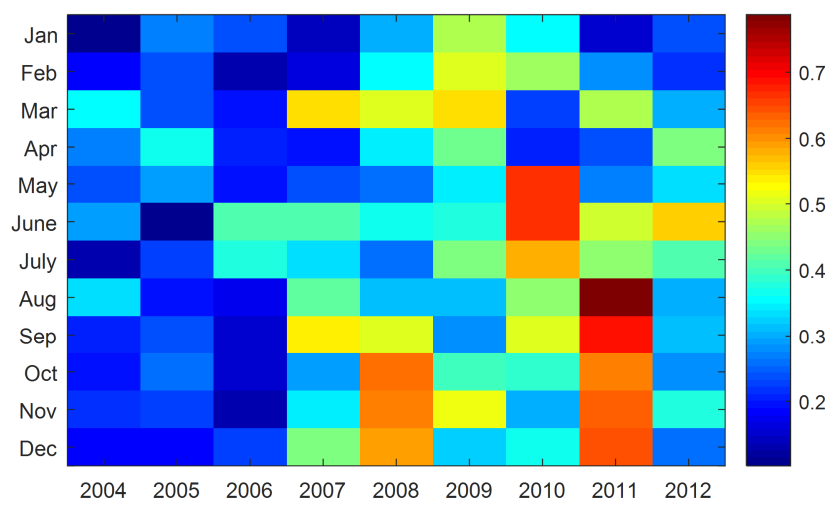

(a) S \& P 500

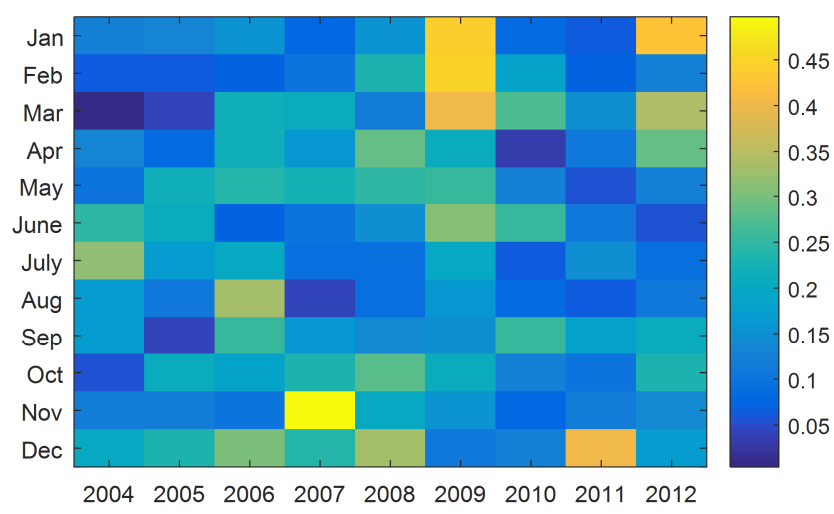

(b) KOSPI

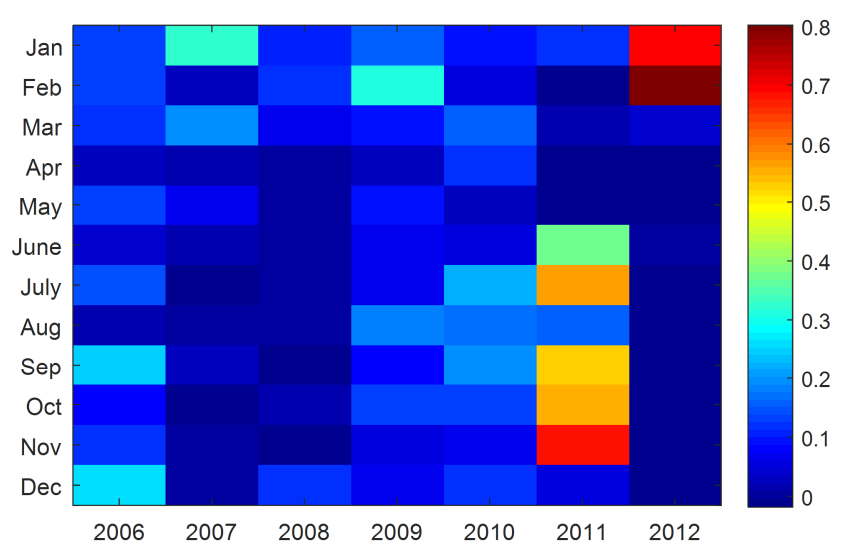

(c) DSE

Figure 5. Impact of index on stock prices.
In contrast, the study observed the low index effect on stock correlations of DSE from January 2006 to mid 2009 with few exceptions which may be due to local crisis generated by political unrest. When, we compare the financial states of S\&P 500 for the years 2007-2008 with the financial states of DSE in the same period, we find that there is no significant effect of DSE index on stock correlations in this period. It implies that global financial crisis did not hit to the DSE in this period. In 2010, there was little effect of index on stock correlations. Although, DSE market crisis has been started from 2010, the severe effect occurred from June 2011 where the remarkable effect of index on stock-stock correlation are found shown by change of color in figure 5 (c). The deep change of color for January and February in 2012 indicates the strong effect of index on stock correlations in these periods. Since, the stiffness of the market is identified by the sharp effect of index on stock-stock correlation, the strong effect of index on stock-stock correlations in January and February imply that the severe change of the market state occurred in 2012.

\section{Conclusion}

The correlations between stocks and index effect on stock correlations of the developed, developing and emerging market have been investigated with evolution of time. At first, we calculate the average correlations between stocks of all markets where we have found that the interactions between stocks become lower before market crash and sharply rise just immediate before the crash. Then, we calculate the average partial correlation of all markets making one month time window. We found that the average partial correlation of the emerging market is significantly higher than those of both developed and developing market except the financial crises. The higher values of partial correlation of the emerging market imply that the index effect on stock correlations of this market is insignificant with the exceptions of crises. During crisis, we have observed lower effect of index on stock-stock correlations in KOSPI 200 than those of other markets. No significant effect of DSE index on stock correlations in the period of global financial crisis implying that global financial crisis did not hit to the DSE in this period. Finally, we identify the market states using our proposed definition which can be useful to understand the market movement of the different types of market located all over the world.

\section{Acknowledgements}

This research was funded by University Grants Commission Bangladesh (UGC- 5921).

\section{References}

[1] Mobarek, A. and Keasey, K. (2000). Weak-form Market Efficiency of an Emerging Market: Evidence from Dhaka Stock Market of Bangladesh. ENBS Conference, 1-30. 
[2] Eom, C., Oh, G. and Kim, S. (2008). Statistical Investigation of Connected Structures of Stock Networks in Financial Time Series. Journal of the Korean Physical Society, 53 (6), 38373841 .

[3] King, B. F. (1996). Market and Industry Factors in Stock Price Behavior. The Journal of Business, 39 (1), 139-190.

[4] Trzcinka, C. (1986). On the Number of Factors in the Arbitrage Pricing Model. The Journal of Finance, 41 (2), 347368

[5] Brown, S. T. (1989). The Number of Factors in Security Returns. The Journal of Finance, 44 (5), 1247-1262.

[6] Gary, C. and Rothschild, M. (1983). Arbitrage and Mean Variance Analyzing the Large Markets. Econometrica, 51, 1281-1301.

[7] Kim, D. and Jeong, H. (2005). Systematic Analysis of Group Identification in Stock Market. Physical Review E, 72, 046133 .

[8] Cai, S. M., Zhou, Y. B., Zhou, T. and Zhou, P. L. (2010). Hierachical Organization and Disassortative Mixing of Correlation-based Weighted Financial Networks. International Journal of Modern Physics C, 21, 433-441.

[9] Gao, Y. C., Wei, Z. W. and Wang, B. H. (2013). Dynamic Evolution of Financial Network and its Relation to Economic Crises. International Journal of Modern Physics C, 24 (02), 1350005.

[10] Markowitz, H. (1952). Portfolio Selection. The Journal of Finance, 7 (1), 77-91.

[11] Kenett, D. Y., Shapira, Y., Madi, A., Bransburg-Zabary, S., Gur- Gershgoren, G. and Ben-Jacob, E. (2011a). Index Cohesive Force Analysis Reveals that the US Market Became Prone to Systemic Collapses Since 2002. PloS one, 6, e19378.

[12] Kenett, Y. N., Kenett, D. Y., Ben-Jacob, E. and Faust, M. (2011b). Global and Local Features of Semantic Networks: Evidence from the Hebrew Mental Lexicon. PloS one, 6, e23912.

[13] Kenett, D. Y., Tumminello, M., Madi, A., Gur- Gershgoren, G., Mantegna, R. N. and Ben-Jacob, E. (2010). Dominating Clasp of the Financial Sector Revealed by Partial Correlation Analysis of the Stock Market. PLoS one, 5, e15032.

[14] Madi, A., Kenett, D., Bransburg-Zabary, S., Merbl, Y., Quintana, F., Boccaletti, S., Tauber, A., Cohen, I. and BenJacob, E., (2011). Analyses of Antigen Dependency Networks Unveil Immune System Reorganization between Birth and Adulthood. Chaos: An Interdisciplinary Journal of Nonlinear Science, 21, 016109-016109.

[15] Jung, Sean S. and Chang, W. (2016). Clustering Stocks using Partial Correlation Coefficients. Physica A, 462, 410-420.
[16] Mantegna, R. N. (1999). Hierarchical Structure in Financial Markets. European Physical Journal B. https://doi.org/10.1007/s100510050929.

[17] Bonanno, G., Caldarelli, G., Lillo, F. and Mantegna, R. N. (2003). Topology of Correlation-based Minimal Spanning Trees in Real and Model Markets. Physical Review E, 68, 046130.

[18] Onnela, J. P., Chakraborti, A., Kaski, K., Kert'esz, J. and Kanto, A. (2003). Dynamics of Market Correlations: Taxonomy and Portfolio Analysis. Physical Review E, 68, 056110 .

[19] Onnela, J. P., Kaski, K. and Kert'esz, J. (2004). Clustering and Information in Correlation based Financial Networks. The European Physical Journal B, March 2004, 38 (2), 353-362.

[20] Kenett, D. Y., Huang, X., Vodenska, I., Havlin, S. and Stanley, H. E. (2015). Partial Correlation Analysis: Applications for Financial Markets. Quantitative Finance, 15 (4), 569-578, DOI: $10.1080 / 14697688.2014 .946660$.

[21] Aste, T., Shaw, W. and Di-Matteo, T. (2010). Correlation Structure and Dynamics in Volatile Markets. New Journal of Physics, 12, 085009.

[22] Baba, K., Shibata, R. and Sibuya, M. (2004). Partial Correlation and Conditional Correlation as Measures of Conditional Independence. Australian \& New Zealand Journal of Statistics, 46, 657-664.

[23] Chakraborty, S. N., Alam, S., Milu, S. A. and Nobi A. (2021). Changes of Network Structure of the Stock Indices of S\&P 500. AIP Conference Proceedings, 2327, 020026.

[24] Shapira, Y., Kenett, D. Y. and Ben-Jacob, E (2009). The Index Cohesive Effect on Stock Market Correlations. The European Physical Journal B, 72, 657-669.

[25] Mantegna, R. N. and Stanley, H. E (2000). An Introduction to Econophysics: Correlation and Complexity in Finance. Cambridge, UK, Cambridge University Press. https://doi.org/10.1063/5.0039405.

[26] Nobi, A. and Lee, J. W. (2017). Systemic Risk and Hierarchical Transitions of Financial Networks. Chaos 27, 063107.

[27] Nobi, A., Lee, S., KIM, D. H. and Lee, J. W. (2014). Correlation and Network Topologies in Global and Local Stock Indices, Physics Letters A, 378 (34), 2482-2489.

[28] Nobi, A., Maeng, S. E., Ha, G. G. and Lee, J. W. (2014). Effects of Global Financial Crisis on Network Structure in Local Stock Market. Physica A, 407, 135-143.

[29] Wang. G., Xie, C., and Stanley, H. E. (2018). Correlation Structure and Evolution of World Stock Market: Evidence from Pearson Partial Correlation Based Networks. Computational Economics, 51 (3): 607-635. DOI: 10.1007/s10614-016-9627-7. 